(2) Open Access Full Text Article

\title{
Decreased apolipoprotein A-I level indicates poor prognosis in extranodal natural killer/T-cell lymphoma, nasal type
}

This article was published in the following Dove Press journal:

OncoTargets and Therapy

14 March 2016

Number of times this article has been viewed

\section{Qi Quan ${ }^{1,2, *}$ \\ Qi Chen ${ }^{1,2, *}$ \\ Ping Chen ${ }^{1,2}$ \\ Li Jiang ${ }^{1,2}$ \\ Tingwei $\mathrm{Li}^{1,2}$ \\ Huijuan Qiu ${ }^{1,2}$ \\ Bei Zhang ${ }^{1-3}$}

'State Key Laboratory of Oncology in South China, ${ }^{2}$ VIP Region,

Sun Yat-Sen University Cancer Center, ${ }^{3}$ Collaborative Innovation

Center of Cancer Medicine, Sun

Yat-Sen University Cancer Center,

Guangzhou, China

*These authors contributed equally to this work
Correspondence: Bei Zhang

VIP Region, Sun Yat-Sen University

Cancer Center, 65I Dongfeng East Road,

Guangzhou, Guangdong, 510060, People's

Republic of China

Tel +862087343455

Fax +86 2087343455

Email zhangbeisysucc@।26.com
Background: Extranodal natural killer (NK)/T-cell lymphoma, nasal type (ENKTL) is an invasive lymphoid malignancy with unfavorable survival, for which a prognostic model has not yet been validated. We hypothesized that serum apolipoprotein A-I (ApoA-I) may serve as a novel prognostic marker for ENKTL.

Patients and methods: A total of 236 newly diagnosed cases of ENKTL were analyzed retrospectively.

Results: The optimal cutoff value for the serum ApoA-I level was determined to be $0.95 \mathrm{~g} / \mathrm{L}$. A total of 154 and 82 cases were assigned to the high and low ApoA-I groups, respectively. Patients in the low ApoA-I group tended to present with poorer clinical features, a lower complete remission rate $(P=0.001)$, and poor median progression-free survival $(P<0.001)$ and overall survival $(P<0.001)$. Multivariate analysis using Cox model showed that the serum ApoA-I level was an independent prognostic marker of overall survival $(P<0.001)$ and progressionfree survival $(P<0.001)$ for ENKTL patients. For cases in the low-risk group, as assessed by International Prognostic Index, Prognosis Index for peripheral T-cell lymphoma, unspecified, and Korean Prognostic Index, the serum ApoA-I level was able to differentiate cases with poor outcomes from cases with good outcomes.

Conclusion: Our results showed that the baseline serum ApoA-I level was helpful for predicting ENKTL prognosis.

Keywords: apolipoprotein A-I, extranodal NK/T-cell lymphoma, prognosis

\section{Introduction}

Extranodal natural killer (NK)/T-cell lymphoma, nasal type (ENKTL) is a predominantly extranodal lymphoma that is related to Epstein-Barr virus (EBV) infection of tumor cells, and it is much more prevalent in Asian and Hispanic countries. ${ }^{1-3}$ ENKTL can emerge with nasal or extranasal involvement, and in $60 \%-90 \%$ of patients, the disease is localized to the upper aerodigestive tract. ${ }^{4,5}$ Generally, radiotherapy is administered for the management of early stage disease, and anthracycline- or asparaginasecontaining chemotherapy followed by radiotherapy is applied to treat advanced cases. However, treatment failures occur frequently in patients with any stage of the disease. ${ }^{6,7}$ Despite radiotherapy and chemotherapy, the survival of ENKTL patients is poor, with less than $50 \%$ of patients surviving at 5 years. , $^{4,8}$

Clinically, the International Prognostic Index (IPI), Korean Prognostic Index (KPI), and Prognosis Index for peripheral T-cell lymphoma, unspecified (PIT) have been applied to predict patient survival and select the optimal therapeutic methods for patients with ENKTL. Past research has shown that $\sim 80 \%$ of patients are categorized 
as low risk based on the IPI model, and some of them exhibit unfavorable survival. ${ }^{9}$ Although PIT can be effectively used to classify other subtypes of T-cell lymphoma, the role of PIT in ENKTL remains controversial. ${ }^{10,11}$ The KPI model has been demonstrated to be more accurate at discriminating different ENKTL subtypes than the IPI and PIT models; however, the prognostic efficacy of KPI was not duplicated in some studies. ${ }^{3}$ In conclusion, the optimal therapy and the prognosis of patients with ENKTL have not yet been well established. Therefore, additional prognostic markers should be developed to improve the stratification of patient outcomes. Several potential prognostic biomarkers, such as absolute lymphocyte count, peripheral blood EBV load, serum C-reactive protein (CRP), and fasting blood glucose level, have been shown to predict the clinical outcomes of patients with ENKTL. ${ }^{12-15}$

As a major protein constituent of high-density lipoprotein cholesterol (HDL-C), apolipoprotein A-I (ApoA-I) is synthesized in the liver and the small intestine. ApoA-I plays a key role in reverse cholesterol transport by transferring cholesterol and phospholipids from peripheral organs to the liver for excretion. ApoA-I also functions as a cofactor of lecithin cholesterol acyltransferase and participates in the turnover of cholesterol to cholesteryl ester. ${ }^{16,17}$ As recently reported, ApoA-I is related to the generation, progression, and prognosis of cancer. ApoA-I has been identified as a potentially useful biomarker for effectively distinguishing cholangiocarcinoma from benign biliary disease and improving the early diagnosis of ovarian cancer. ${ }^{18,19}$ Moreover, decreased serum ApoA-I has been shown to be correlated with worse overall survival (OS) in lung cancer and metastatic nasopharyngeal carcinoma. ${ }^{20,21}$ The role of ApoA-I in patients with ENKTL has not yet been reported. In our clinical observation, we found that patients with lower baseline ApoA-I tended to present with a shorter survival time. Therefore, we retrospectively analyzed the prognostic value of baseline serum ApoA-I in patients with ENKTL.

\section{Patients and methods}

\section{Patient selection}

We included 236 patients pathologically diagnosed with ENKTL from June 2002 to May 2014 at the Sun Yat-Sen University Cancer Center in this study. Cases were included if they met the following criteria: 1) histologically confirmed diagnosis of ENKTL based on the guidelines of the World Health Organization; 2) NK/T-cell type, as proven by immunohistochemistry or flow cytometry findings; 3 ) no previous tumor-related treatment; and 4) complete medical record and follow-up information. Patients were excluded if they suffered from acute illnesses, including stroke, acute infection, surgery, and trauma. This retrospective study was approved by the Institutional Review Board of Sun Yat-Sen University Cancer Center and by the Ethics Committees of Sun Yat-Sen University Cancer Center.

\section{Clinical data collection and staging}

The following data were collected at diagnosis: patient demographics; medical examination results; weight; height; serum lactate dehydrogenase (LDH); serum beta-2 microglobulin $(\beta 2 \mathrm{M})$; serum lipids and lipoproteins (total cholesterol, triglyceride, HDL-C, low-density lipoprotein cholesterol [LDL-C], ApoA-I, and apolipoprotein-B [ApoB]); CRP; results of marrow tests, results of endoscopic examination of the nasal and oral cavities; and computed tomography or magnetic resonance scans of the nasopharynx, neck, chest, and abdominopelvic or positron emission tomography/computed tomography of the full body. For all cases, the plasma levels of lipids and lipoproteins in the fasting state were determined by an automatic biochemical analyzer. Prior to use of the patients' sera, written informed consent was obtained from each of the participants.

ENKTL is classified into two categories depending on the site of the lesions: 1) upper aerodigestive tract NK/T-cell lymphoma (UNKTL), defined as a case with the primary tumor located in the nasal cavity, nasopharynx, or upper aerodigestive tract and 2) extra-upper aerodigestive tract NK/T-cell lymphoma (EUNKTL), defined as a case with the primary tumor located at any site other than UNKTL sites. Although these two subclasses share the same pathological characteristics, UNKTL and EUNKTL have obviously different prognoses. ${ }^{5,22}$ A standard staging system for NK/T-cell lymphomas is lacking. We staged all cases using the Ann Arbor staging system, which was originally designed for Hodgkin's lymphoma. IPI (performance status [PS], age, extranodal sites, serum LDH, and stage), KPI (B symptoms, regional lymph nodes, serum $\mathrm{LDH}$, and stage), and PIT (PS, age, bone marrow involvement, and serum LDH) were also evaluated to examine patient survival. ${ }^{3,10,23}$

\section{Response assessment and statistical analyses}

We utilized the International Working Group Recommendations for Response Criteria for non-Hodgkin's lymphoma (NHL) to evaluate the reactions of the ENKTL patients to the administered therapies. ${ }^{24}$ Regular imaging surveillance was performed every 3 months for the first 2 years after treatment, twice a year for the next 3 years, and annually or when clinical signs indicated it thereafter. No pellucid marginal value 
for serum lipids or lipoproteins was found to be associated with tumor outcome, so we analyzed the receiver operating characteristic (ROC) curve to provide an optimal critical value for ApoA-I and ApoB. Based on the National Cholesterol and Education Program Adult Treatment Panel III criteria, a total cholesterol serum concentration $\geq 200 \mathrm{mg} / \mathrm{dL}$, a triglyceride serum concentration $\geq 150 \mathrm{mg} / \mathrm{dL}$, a HDL-C serum concentration $<40 \mathrm{mg} / \mathrm{dL}$, and a LDL-C serum concentration $\geq 130 \mathrm{mg}$ / $\mathrm{dL}$ are defined as hypercholesterolemia, hypertriglyceridemia, categorical low HDL-C, and high LDL-C, respectively. ${ }^{25}$

Statistical analyses were conducted using SPSS standard version 20.0 (IBM Corporation, Armonk, NY, USA) for Windows. OS was defined as the interval between the diagnosis date and the time of death for any reason or last follow-up. Progression-free survival (PFS) was determined from the date of diagnosis to the time of disease progression, relapse after response, last follow-up, or death. Differences in continuous variables were analyzed by nonparametric tests (Mann-Whitney $U$-test or Kruskal-Wallis). Categorical characteristics were compared by the chi-square test. Survival curves were analyzed by the Kaplan-Meier method and the log-rank test was used to compare differences between groups. The multivariate Cox regression model was used to evaluate independent predictive factors associated with survival difference. A two-tailed $P$-value of $<0.05$ was considered statistically significant.

\section{Results}

The clinical baseline features of the 236 cases are listed in Table 1 . The median age at diagnosis was 41 years (range: $16-78$ years), and $66.5 \%$ of the patients were male. The majority of cases originally manifested as the UNKTL subtype ( $\mathrm{n}=198,83.9 \%$ ). For the EUNKTL cases, the primary tumor was mainly located in the skin, gastrointestinal tract, testis, and spleen. A total of 221 patients (93.6\%) had a favorable PS. More than half of the patients showed B symptoms. A total of 83 cases (35.2\%) exhibited increased LDH. Of the 146 patients for whom $\beta 2 \mathrm{M}$ was measured, 82 (56.9\%) showed increased $\beta 2 \mathrm{M}$. A total of $130(55.1 \%)$ and $123(52.1 \%)$ patients presented with regional lymph node involvement and two or more extranodal sites, respectively. Only eight patients (3.4\%) displayed bone marrow infiltration. Most cases presented with localized disease (Ann Arbor stages I/II; $n=185,78.4 \%$ ). According to the IPI score, 151 patients $(64.0 \%)$ with less than two adverse factors were categorized in the low-risk group. The KPI assessment classified 120 patients $(50.8 \%)$ into the low-risk group. The PIT model classified 215 patients $(91.1 \%)$ into the low-risk group, and 21 patients $(8.1 \%)$ exhibited two or more adverse factors.
Correlation between serum ApoA-I and clinical features

The median serum ApoA-I level (range) and 25\% and 75\% quartiles were $1.06(0.20-1.80 \mathrm{~g} / \mathrm{L}), 0.89$, and $1.26 \mathrm{~g} / \mathrm{L}$, respectively. The optimal cutoff value for serum ApoA-I was $0.95 \mathrm{~g} / \mathrm{L}$ based on the ROC analysis results (area under the curve: $0.719,95 \%$ confidence interval (CI): 0.657-0.775, $P<0.001$ ) (Figure 1). The prognostic value of the different cutoff values, including median value $(1.06 \mathrm{~g} / \mathrm{L})$ and mean value $(1.07 \mathrm{~g} / \mathrm{L})$, were also evaluated, and $0.95 \mathrm{~g} / \mathrm{L}$ was found to be the most effective cutoff value. The whole cohort was divided into two groups based on the $0.95 \mathrm{~g} / \mathrm{L}$ cutoff value. One hundred and fifty-four (65.3\%) cases were categorized into the high ApoA-I group, and the remaining 82 (34.7\%) patients were categorized into the low ApoA-I group (Table 1). The optimal cutoff value for ApoB was $0.88 \mathrm{~g} / \mathrm{L}$. The baseline features of the cases with ApoA-I $\leq 0.95 \mathrm{~g} / \mathrm{L}$ were compared with those of cases with ApoA-I $>0.95 \mathrm{~g} / \mathrm{L}$ (Table 1). Poor PS, B symptoms, elevated serum $\mathrm{LDH}$, elevated serum $\beta 2 \mathrm{M}$, and advanced stage (III/IV) occurred more frequently in the cases from the low ApoA-I group. Patients in the low ApoA-I group tended to show a higher rate of hypercholesterolemia, decreased HDL-C, hypoalbuminemia, and increased CRP. Additionally, the patients in low ApoA-I group exhibited a higher risk than those in the high ApoA-I group according to the IPI, KPI, and PIT models. The continuous variable analyses showed that the ApoA-I level was notably lower in patients with EUNKTL and two or more extranodal sites. No significant intergroup differences in the mean values of continuous or categorical variables were found for any other clinical features examined.

\section{Treatment and response}

The therapeutic protocols and responses are summarized in Table 2. Chemotherapy followed by radiotherapy was administered to 148 (62.7\%) patients, chemotherapy alone was administered to $66(28.0 \%)$ patients, radiotherapy alone was administered to four (1.7\%) patients, surgery followed by chemotherapy was administered to 16 patients $(6.8 \%)$, and best supportive care alone was administered to two cases $(0.8 \%)$. No significant differences in treatment modalities were observed between the high and low ApoA-I groups.

The first-line chemotherapy schemes were as follows: cyclophosphamide, doxorubicin, vincristine, and prednisone $(\mathrm{CHOP})$ or CHOP-like (CHOP + L-asparaginase, $\mathrm{CHOP}+$ high-dose methotrexate, or CHOP + etoposide); alternating triple therapy (cyclophosphamide, doxorubicin, vincristine, prednisone, and bleomycin, ifosfamide, methotrexate, 
Table I Baseline characteristics of patients by serum ApoA-I level

\begin{tabular}{|c|c|c|c|c|c|}
\hline Characteristics & $\begin{array}{l}\text { ApoA-I value } \\
(\text { mean } \pm \text { SD) }(g / L)\end{array}$ & $P$-value & $\begin{array}{l}\text { Low ApoA-I } \\
\text { group, n (\%) }\end{array}$ & $\begin{array}{l}\text { High ApoA-I } \\
\text { group, n (\%) }\end{array}$ & $P$-value \\
\hline Number of cases & & & $82(34.7)$ & $154(65.3)$ & \\
\hline Age at diagnosis (years) & & 0.141 & $4 I(16-78)$ & $38(16-73)$ & 0.297 \\
\hline$\leq 60$ & $1.08 \pm 0.27$ & & $69(84.1)$ & $138(89.6)$ & \\
\hline$>60$ & $1.01 \pm 0.22$ & & $13(15.9)$ & $16(10.4)$ & \\
\hline Sex & & 0.319 & & & 0.668 \\
\hline Male & $1.06 \pm 0.27$ & & $55(67.1)$ & $102(66.2)$ & \\
\hline Female & $1.10 \pm 0.27$ & & $27(32.9)$ & $52(33.8)$ & \\
\hline ECOG PS & & $<0.001$ & & & 0.003 \\
\hline 0,1 & $1.09 \pm 0.26$ & & $71(91.5)$ & $150(96.1)$ & \\
\hline$\geq 2$ & $0.8 \mathrm{I} \pm 0.27$ & & II (8.5) & $4(3.9)$ & \\
\hline Subtypes & & 0.006 & & & 0.094 \\
\hline UNKTL & $1.09 \pm 0.26$ & & $64(78.0)$ & $134(87.0)$ & \\
\hline EUNKTL & $0.96 \pm 0.31$ & & $18(22.0)$ & $20(13.0)$ & \\
\hline B symptoms & $1.0 \mathrm{I} \pm 0.28$ & $<0.00$ I & $53(64.6)$ & $67(43.5)$ & 0.003 \\
\hline Regional lymph node involvement & $1.03 \pm 0.27$ & 0.057 & $52(63.4)$ & $78(50.6)$ & 0.074 \\
\hline Extranodal sites $\geq 2$ & $1.03 \pm 0.28$ & 0.003 & $50(61.0)$ & $73(47.4)$ & 0.056 \\
\hline Bone marrow involvement & $0.94 \pm 0.28$ & 0.126 & $5(6.1)$ & $3(1.9)$ & 0.130 \\
\hline Elevated serum LDH & $0.99 \pm 0.26$ & 0.001 & $38(46.3)$ & $45(29.2)$ & 0.010 \\
\hline Elevated serum $\beta 2 \mathrm{M}^{*}$ & $0.98 \pm 0.28$ & $<0.001$ & $4 \mathrm{I}(50.0)$ & $4 \mathrm{I}(44.6)$ & 0.001 \\
\hline Ann Arbor stage & & $<0.00$ I & & & $<0.001$ \\
\hline $\mathrm{I} / \mathrm{II}$ & $1.11 \pm 0.26$ & & $52(63.4)$ & I 33 (86.4) & \\
\hline III/IV & $0.94 \pm 0.27$ & & $30(36.6)$ & $21(13.6)$ & \\
\hline IPI score & & $<0.001$ & & & 0.010 \\
\hline $0-1$ & $1.14 \pm 0.26$ & & $43(52.4)$ & $108(70.1)$ & \\
\hline $2-5$ & $0.95 \pm 0.24$ & & $39(47.6)$ & $46(29.9)$ & \\
\hline KPI score & & $<0.001$ & & & 0.004 \\
\hline 0-I & $1.13 \pm 0.26$ & & $31(37.8)$ & $89(57.8)$ & \\
\hline $2-4$ & $1.01 \pm 0.27$ & & $51(62.2)$ & $65(42.2)$ & \\
\hline PIT score & & 0.032 & & & 0.031 \\
\hline $0-1$ & $1.09 \pm 0.26$ & & $70(85.4)$ & I 45 (94.2) & \\
\hline $2-4$ & $0.95 \pm 0.29$ & & $12(14.6)$ & $9(5.8)$ & \\
\hline Cholesterol ( $\geq 200 \mathrm{mg} / \mathrm{dL})$ & $1.21 \pm 0.26$ & $<0.001$ & II (13.4) & $51(33.1)$ & 0.001 \\
\hline Triglyceride ( $\geq 150 \mathrm{mg} / \mathrm{dL})$ & $I .1 I \pm 0.33$ & 0.334 & $28(34.1)$ & $46(29.9)$ & 0.556 \\
\hline HDL-C $(<40 \mathrm{mg} / \mathrm{dL})$ & $0.90 \pm 0.21$ & $<0.001$ & $68(82.9)$ & $39(25.3)$ & $<0.001$ \\
\hline LDL-C $>2.75(\geq 130 \mathrm{mg} / \mathrm{dL})$ & $1.21 \pm 0.30$ & 0.058 & $16(19.5)$ & $43(27.9)$ & 0.206 \\
\hline ApoB $(\geq 0.88 \mathrm{~g} / \mathrm{L})$ & $1.13 \pm 0.34$ & 0.765 & $31(37.8)$ & $35(22.7)$ & 0.633 \\
\hline Albumin $(<35 \mathrm{~g} / \mathrm{L})$ & $0.93 \pm 0.37$ & $<0.001$ & $20(24.2)$ & $12(7.8)$ & 0.001 \\
\hline $\operatorname{CRP}(>10 \mathrm{mg} / \mathrm{L})$ & $1.00 \pm 0.30$ & $<0.001$ & $60(73.2)$ & $36(25.0)$ & $<0.001$ \\
\hline BMI $\left(\geq 25 \mathrm{~kg} / \mathrm{m}^{2}\right)$ & $1.01 \pm 0.20$ & 0.251 & $9(I I .0)$ & $18(11.7)$ & 1.000 \\
\hline
\end{tabular}

Note: *Serum $\beta 2 M$ was measured in 146 patients.

Abbreviations: ApoA-I, apolipoprotein A-I; ApoB, apolipoprotein-B; $\beta 2 \mathrm{M}$, beta-2 microglobulin; BMI, body mass index; CRP, C-reactive protein; ECOG PS, Eastern Cooperative Oncology Group performance status; EUNKTL, extra-upper aerodigestive tract NK/T-cell lymphoma; HDL-C, high-density lipoprotein cholesterol; IPI, International Prognostic Index; KPI, Korean Prognostic Index; LDH, lactate dehydrogenase; LDL-C, low-density lipoprotein cholesterol; PIT, Prognosis Index for peripheral T-cell lymphoma, unspecified; SD, standard deviation; UNKTL, upper aerodigestive tract NK/T-cell lymphoma.

etoposide, and dexamethasone, cisplatin, and cytarabine); etoposide, doxorubicin, vincristine, cyclophosphamide, and prednisone; oxaliplatin and gemcitabine; oxaliplatin, gemcitabine, and L-asparaginase; and dexamethasone, methotrexate, ifosfamide, L-asparaginase, and etoposide. The patients were administered two to eight courses of initial chemotherapy. At our institute, a total radiotherapy dose of 30-60 Gy with fractional doses of 1.8-2.0 Gy is generally used. Two hundred and twenty-six patients $(95.8 \%)$ were evaluated to determine their responses to initial chemotherapy, and 104 of these 226 patients $(46.0 \%)$ exhibited a complete response (CR) or unconfirmed CR, 88 patients (38.9\%) achieved a partial response, 17 patients (7.5\%) exhibited stable disease, and 17 patients $(7.5 \%)$ showed progressive disease; therefore, the overall response rate (ORR) was $85.0 \%$. The $\mathrm{CR}$ rate and ORR to original treatment were significantly higher in the 


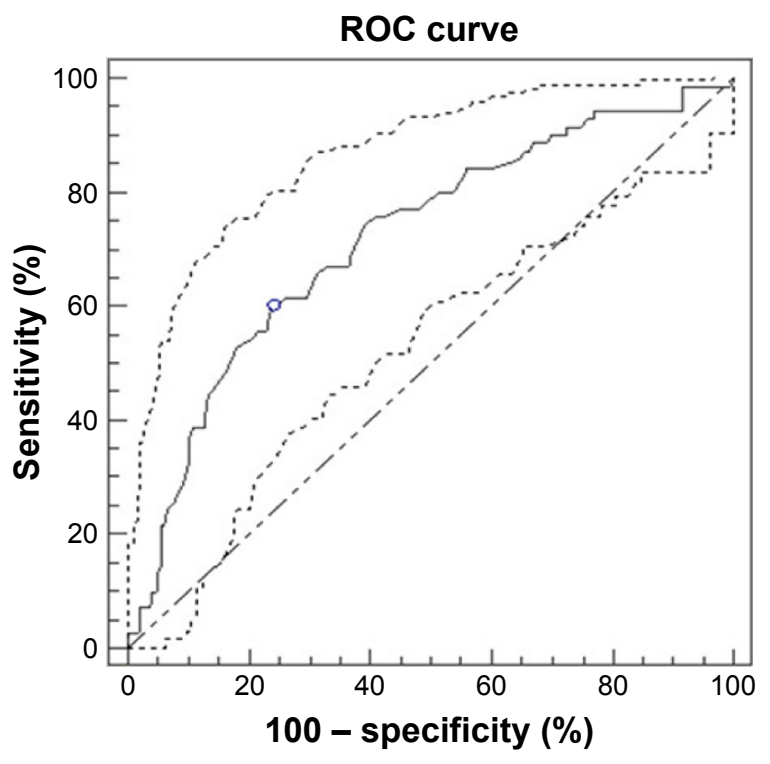

Figure I ROC of pretreatment ApoA-I level for outcome prediction. Notes: Area under the ROC curve: 0.719 ( $95 \% \mathrm{Cl}: 0.657-0.775)$. The sensitivity and specificity of the point with highest accuracy were $60 \%$ and $75.9 \%$, respectively. Abbreviations: ApoA-I, apolipoprotein A-I; Cl, confidence interval; ROC, receiver operating curve.

high ApoA-I group than in the low ApoA-I group (54.1\% vs $30.8 \%, P=0.001 ; 90.5 \%$ vs $74.4 \%, P=0.005$, respectively).

\section{Survival analysis}

The cutoff point for follow-up data collection was April 12, 2015, and the median follow-up duration was 27.3 months (range: 2.2-154.2 months). In the current study, the 3-year OS and PFS of the patients were $67.0 \%$ and $55.6 \%$, respectively. The patients from the low ApoA-I group exhibited lower PFS (3-year PFS, 66.1\% vs 34.4\%; $P<0.001$ ) and OS (3-year OS, $80 \%$ vs $41.0 \% ; P<0.001$; Figure 2) than the patients in the high ApoA-I group. The univariate and multivariate analyses of OS and PFS of the 236 patients are presented in Table 3. In the univariate analysis, poor PS, EUNKTL, B symptoms, bone marrow involvement, two or more extranodal sites, regional lymph node involvement, increased serum LDH, increased serum $\beta 2 \mathrm{M}$, hypertriglyceridemia, hypoalbuminemia, decreased HDL-C, decreased ApoA-I, advanced stage, and the IPI, PIT, and KPI values were dramatically correlated with poor OS and PFS. Age was significantly correlated with $\mathrm{OS}(P=0.012)$, but failed to demonstrate prognostic significance for PFS $(P=0.080)$. The clinical factors that were statistically significant predictors of OS and PFS were included in the multivariate analysis. IPI, KPI, and PIT scores were not included in the multivariate analysis because these scores overlapped with other clinical variables. The multivariate analysis results revealed that the serum ApoA-I level was an independent prognostic indicator of OS (relative risk: 3.709, 95\% CI: 1.908-7.210, $P<0.001$ ) and PFS (relative risk: 2.669, 95\% CI: 1.536-4.640, $P=0.001$ ). Additionally, poor PS, EUNKTL, and marrow involvement were considered independent prognostic indicators of OS and PFS.

\section{Discussion}

The current study showed that a low level of serum ApoA-I was significantly correlated with inferior OS and PFS in patients with ENKTL. In addition to low ApoA-I, poor PS, EUNKTL, and marrow infiltration were independent prognostic indicators of OS and PFS, which is consistent with the findings of previous studies. ${ }^{26-29}$

The mechanisms underlying the relationship between decreased ApoA-I levels and short survival time have

Table 2 Primary treatment and response in patients with extranodal natural killer (NK)/T-cell lymphoma

\begin{tabular}{|c|c|c|c|}
\hline Treatment & $\begin{array}{l}\text { Low ApoA-I group } \\
\text { (number of patients) }\end{array}$ & $\begin{array}{l}\text { High ApoA-I group } \\
\text { (number of patients) }\end{array}$ & $P$-value \\
\hline Patients treated & 81 & 153 & 0.649 \\
\hline Treatment modalities & & & 0.401 \\
\hline CT followed by RT & 51 & 97 & \\
\hline CT alone & 22 & 44 & \\
\hline RT alone & 0 & 4 & \\
\hline Surgery followed by $\mathrm{CT}$ & 8 & 8 & \\
\hline Best supportive care & I & I & \\
\hline Chemotherapy regimens & & & 0.826 \\
\hline Adriamycin-used & 32 & 57 & \\
\hline L-asparaginase-used & 36 & 67 & \\
\hline Both & 7 & 11 & \\
\hline Efficacy* & & & 0.001 \\
\hline CR achieved & 24 & 80 & \\
\hline
\end{tabular}

Note: *The response to treatment was evaluated in 226 patients.

Abbreviations: ApoA-I, apolipoprotein A-I; CR, complete remission; CT, chemotherapy; RT, radiotherapy. 
A

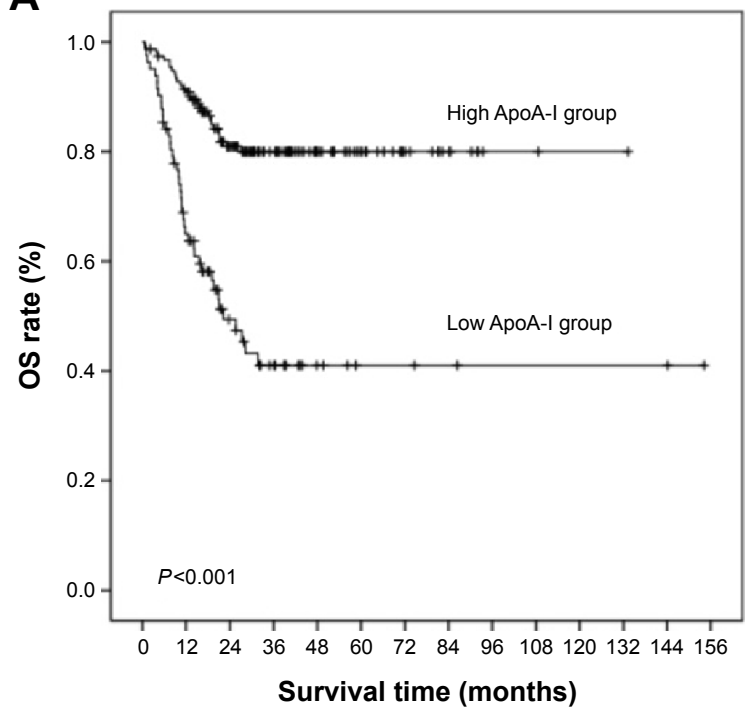

B

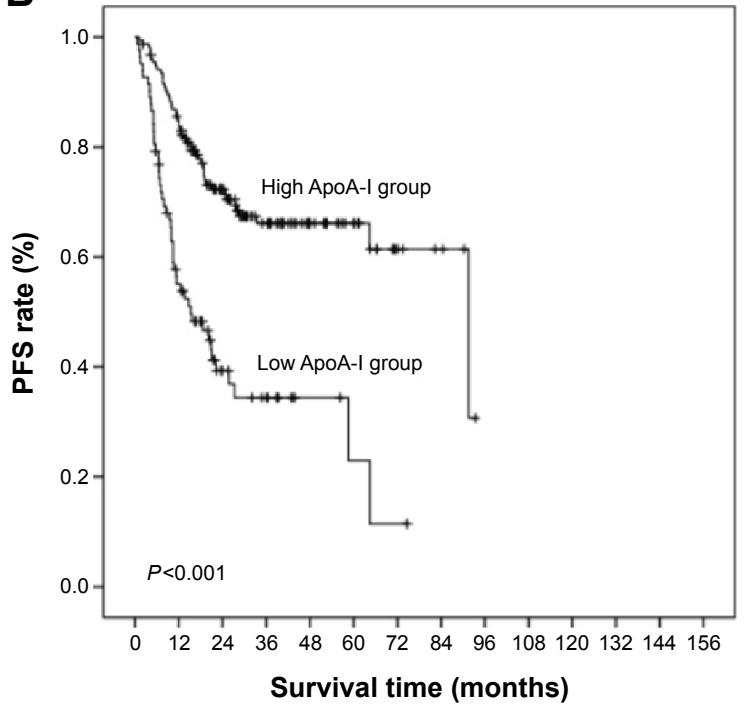

Figure 2 OS and PFS according to ApoA-I level ( $\leq 0.95$ vs $>0.95$ g/L). Kaplan-Meier plots of OS (A) and PFS (B) for all patients.

Abbreviations: ApoA-I, apolipoprotein A-I; OS, overall survival; PFS, progression-free survival.

not been fully elucidated; nevertheless, several potential interpretations have been suggested. First, patients with a low ApoA-I level may have enhanced tumor cell growth and metastatic ability. Chronic inflammation has been shown to be associated with various steps involved in tumorigenesis and development. ${ }^{30,31}$ A recent report by Lin et $\mathrm{al}^{27}$ found that ENKTL patients have a multitude of tumor-associated macrophages, which is one of the most vital actors in the inflammation arena and has been accepted as a crucial marker of inflammation. An increasing number of studies have

Table 3 Results of univariate and multivariate analyses of prognostic factors for PFS and OS in patients with ENKTL

\begin{tabular}{|c|c|c|c|c|c|c|}
\hline \multirow[t]{3}{*}{ Parameter } & \multicolumn{3}{|l|}{ PFS } & \multicolumn{3}{|l|}{ OS } \\
\hline & \multirow{2}{*}{$\begin{array}{l}\text { Univariate } \\
\text { analysis } \\
P \text {-value }\end{array}$} & \multicolumn{2}{|c|}{ Multivariate analysis } & \multirow{2}{*}{$\begin{array}{l}\text { Univariate } \\
\text { analysis } \\
P \text {-value }\end{array}$} & \multicolumn{2}{|c|}{ Multivariate analysis } \\
\hline & & RR (95\% Cl) & $P$-value & & $\operatorname{RR}(95 \% \mathrm{CI})$ & $P$-value \\
\hline Age $>60$ years & 0.080 & & & 0.011 & & \\
\hline ECOG PS $\geq 2$ & $<0.001$ & $2.893(1.350-6.200)$ & 0.006 & $<0.001$ & $2.618(1.202-5.703)$ & 0.015 \\
\hline Subtype, EUNKTL & $<0.001$ & $2.690(1.537-4.706)$ & 0.001 & $<0.001$ & $2.346(1.233-4.464)$ & 0.009 \\
\hline B symptoms & 0.003 & & & $<0.00 \mathrm{I}$ & & \\
\hline Bone marrow involvement & $<0.001$ & $3.699(1.415-9.668)$ & 0.008 & $<0.001$ & $3.152(1.115-8.913)$ & 0.030 \\
\hline Extranodal sites $\geq 2$ & $<0.001$ & & & 0.001 & & \\
\hline $\begin{array}{l}\text { Regional lymph node } \\
\text { involvement }\end{array}$ & 0.002 & & & 0.001 & & \\
\hline LDH $(>245$ U/L) & $<0.001$ & & & $<0.001$ & & \\
\hline$\beta 2 \mathrm{M}^{*}(>2.52 \mathrm{mg} / \mathrm{L})$ & $<0.001$ & & & $<0.001$ & & \\
\hline Stages III-IV & $<0.00$ I & & & $<0.001$ & & \\
\hline Cholesterol ( $\geq 200 \mathrm{mg} / \mathrm{dL})$ & 0.132 & & & 0.212 & & \\
\hline Triglyceride ( $\geq 150 \mathrm{mg} / \mathrm{dL})$ & 0.012 & & & 0.032 & & \\
\hline HDL-C (<40 mg/dL) & 0.009 & & & 0.043 & & \\
\hline LDL-C ( $\geq 130$ mg/dL) & 0.878 & & & 0.429 & & \\
\hline ApoA-I $(<0.95 \mathrm{~g} / \mathrm{L})$ & $<0.001$ & $2.669(1.536-4.640)$ & 0.001 & $<0.001$ & $3.709(1.908-7.210)$ & $<0.001$ \\
\hline ApoB ( $\geq 0.88 \mathrm{~g} / \mathrm{L})$ & 0.523 & & & 0.653 & & \\
\hline Albumin $(<35.0 \mathrm{~g} / \mathrm{L})$ & $<0.001$ & & & $<0.001$ & & \\
\hline CRP $(\geq 10.0 \mathrm{mg} / \mathrm{L})$ & 0.151 & & & 0.086 & & \\
\hline $\mathrm{BMI}\left(\geq 25 \mathrm{~kg} / \mathrm{m}^{2}\right)$ & 0.570 & & & 0.507 & & \\
\hline
\end{tabular}

Note: *Serum $\beta 2 M$ was measured in 146 patients.

Abbreviations: ApoA-I, apolipoprotein A-I; ApoB, apolipoprotein-B; $\beta 2 \mathrm{M}$, beta-2 microglobulin; BMI, body mass index; Cl, confidence interval; CRP, C-reactive protein; ECOG PS, Eastern Cooperative Oncology Group performance status; ENKTL, extranodal natural killer (NK)/T-cell lymphoma, nasal type; EUNKTL, extra-upper aerodigestive tract NK/T-cell lymphoma; HDL-C, high-density lipoprotein cholesterol; LDH, lactate dehydrogenase; LDL-C, low-density lipoprotein cholesterol; OS, overall survival; PFS, progression-free survival; RR, relative risk. 
suggested that ApoA-I can potently inhibit tumor development through an expansive repertoire of activities, including anti-inflammatory, antiangiogenic, antithrombotic, and immune-regulatory activities, but not anti-atherosclerotic activities. ${ }^{17,32-34}$ A common mechanism of action of the anti-inflammatory effect of ApoA-I is its ability to bind to proinflammatory phospholipids. ${ }^{32,35}$ Moreover, ENKTL is associated with EBV infection, and latent membrane protein 1 encoded by EBV has been reported to promote interleukin-6 production. ${ }^{2,36}$ Interleukin- 6 and tumor necrosis factor- $\alpha$ can decrease the synthesis and secretion rates of ApoA-I by hepatic cells, and a low ApoA-I level indirectly indicates increased cytokine release and a stronger inflammatory response to tumors. ${ }^{37}$ Prostacyclin (PGI2) stabilization is considered an important function of ApoA-I. When the ApoA-I level is reduced, the availability of PGI2 at the location of vascular endothelial injury can be reduced, thereby decreasing the protection against thrombocyte aggregation and thrombosis, which is strongly related to tumor cell growth and metastasis. ${ }^{17,38}$ Second, patients with low ApoA-I have been proposed to have a poorer response to treatment, and this hypothesis was supported by our results, which demonstrated that the $\mathrm{CR}$ rate and $\mathrm{ORR}$ in patients with low ApoA-I were significantly inferior to those of patients with high ApoA-I, though no remarkable difference in primary therapeutic strategy was observed between those groups. A possible explanation for this finding is that patients with low ApoA-I may be less tolerant of chemoradiotherapy, which results in the administration of an insufficient dose of chemotherapy or radiotherapy. Third, patients with lower ApoA-I levels had more adverse clinical characteristics, such as poor PS, advanced stage, and B symptoms.

Here, we retrospectively analyzed the relationship between the serum ApoA-I level and clinical characteristics and discovered that low ApoA-I level was more frequent in cases with elevated $\mathrm{LDH}$, elevated $\beta 2 \mathrm{M}$, elevated $\mathrm{CRP}$, and decreased albumin. Serum LDH and $\beta 2 \mathrm{M}$ levels are indicators of tumor burden in patients with ENKTL and suggest that ApoA-I is a potential marker of tumor load to a certain extent. ${ }^{39,40} \mathrm{~A}$ prior study by Li et al observed that high CRP level is associated with unsatisfactory outcomes in ENKTL. ${ }^{14}$ Hypoalbuminemia is associated with nutritional deficiency and body weight reduction, which result in a poor PS and increased cancerrelated mortality. ${ }^{41}$ The results of our univariate survival analyses revealed that elevated $\mathrm{LDH}$, elevated $\beta 2 \mathrm{M}$, and low serum albumin were significantly related to PFS and OS in patients with ENKTL. Inconsistent with previous investigations, our results showed that low HDL-C and high CRP were not significantly correlated with poor clinical outcome when other serum lipids and lipoproteins and clinical characteristics were included in the analysis. ${ }^{14,42}$ These discrepancies may be associated with selection criteria. For example, Wang et $\mathrm{al}^{42}$ included patients who received either oxaliplatin, gemcitabine, L-asparaginase or etoposide, doxorubicin, vincristine, cyclophosphamide, and prednisone regimens as induction chemotherapy. In addition, the patients with lower ApoA-I levels in our study had other adverse clinical characteristics, including poor PS, advanced stage, and B symptoms, all of which could be responsible for the relationship between decreased serum ApoA-I level and poor prognosis.

We performed univariate analyses of the prognostic values of the IPI, PIT, and KPI models, and the three models were found to have high prognostic value. However, the IPI and PIT prognostic models disproportionately categorized the patients into the low-risk category, with the models categorizing $64.0 \%$ and $91.1 \%$ of the patients into the low-risk category, respectively (Table 1). Although the KPI model equally distributed patients into the different risk categories, all three models failed to distinguish cases within the low-risk group that had poor clinical outcomes. As shown in Figure 3, serum ApoA-I can be used to divide low-risk cases into two subgroups with different prognoses. In our study, the proportion of low-risk IPI scores was less than that of previous reports due to patient selection criteria. ${ }^{3,4,12}$

As described in the "Introduction", optimal treatment strategies for ENKTL have not been fully defined, and novel drugs for ENKTL should be developed. Recently, some studies suggested that the pharmacological administration of ApoA-I may have a therapeutic benefit as an anticancer agent in melanoma. ApoA-I mimetic peptides engineered to mimic anti-inflammatory and antioxidant functionalities of ApoA-I have recently been reported to suppress ovarian cancer cell growth. ${ }^{43,44}$ Although these findings have not yet been successfully confirmed in the clinical context, we expect that ApoA-I and its mimetic peptides will be valuable therapeutic agents to complement antitumor strategies in ENKTL in the near future. We acknowledge that the present study is limited due to its retrospective, single-center design. Some patients with cardiovascular complaints or hepatic illnesses, such as coronary heart disease, hypertension, and chronic hepatitis, were included in this study, and the effects of those disease on lipid metabolism were not considered.

\section{Conclusion}

Our study is first to demonstrate that baseline serum ApoA-I can function as a strong and independent indicator of survival outcomes in ENKTL. This biomarker is directly derived from routine blood biochemical tests and can be easily measured in the clinic. Nevertheless, more studies with multicenter or 
A

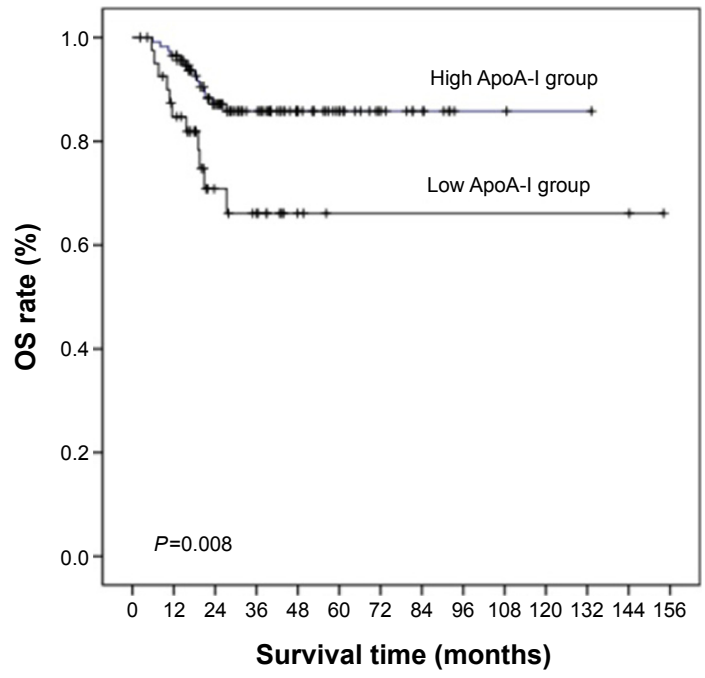

C

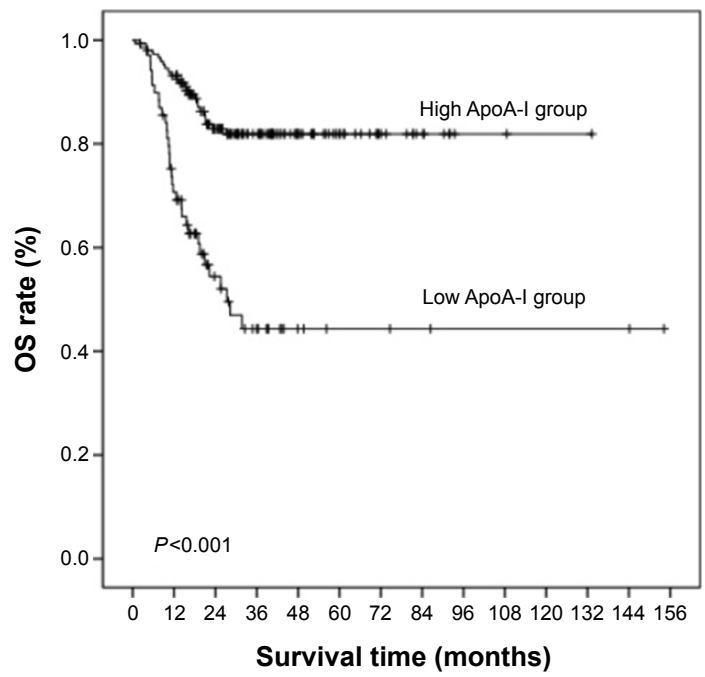

E

KPI score $=0-1$

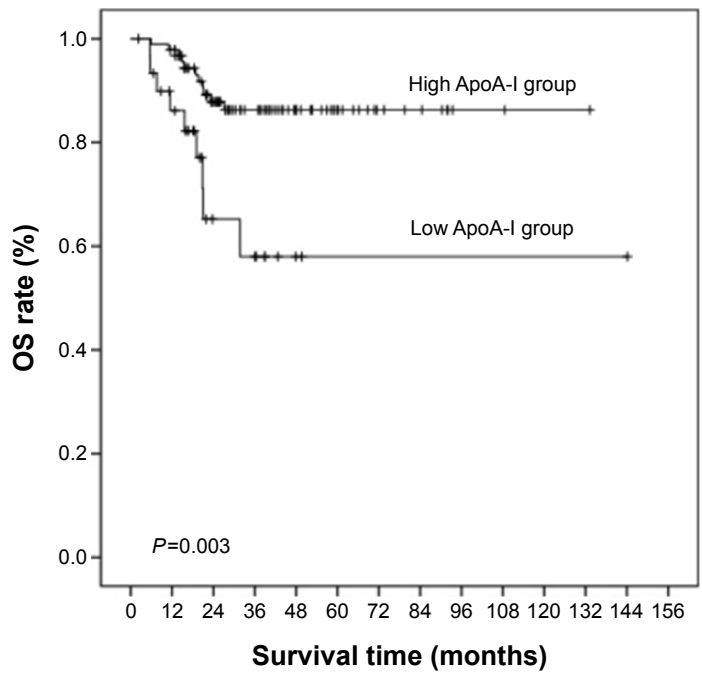

B

IPI score $=0-1$

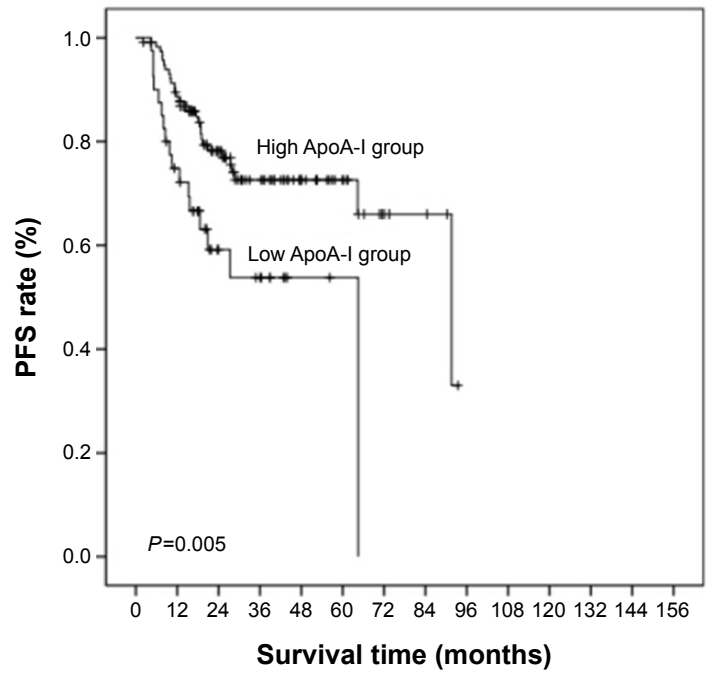

D

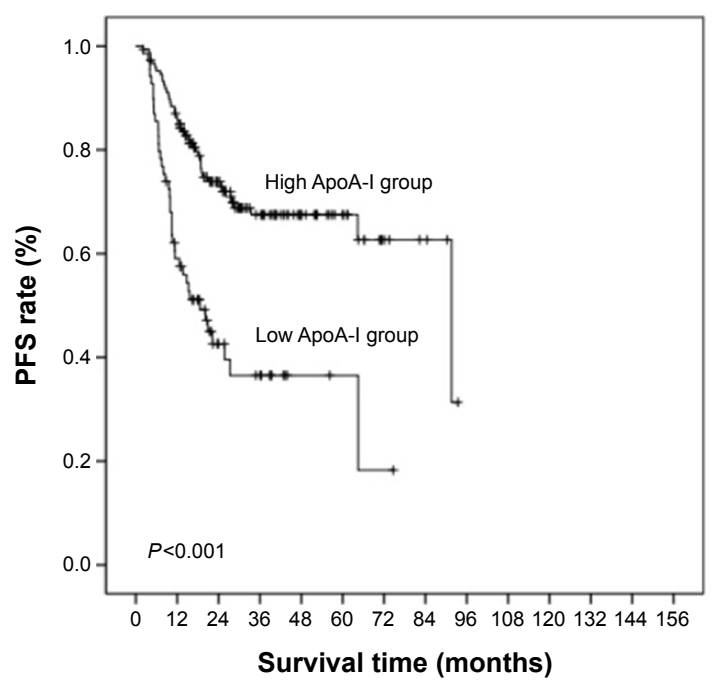

$\mathbf{F}$

$\mathrm{KPI}$ score $=0-1$

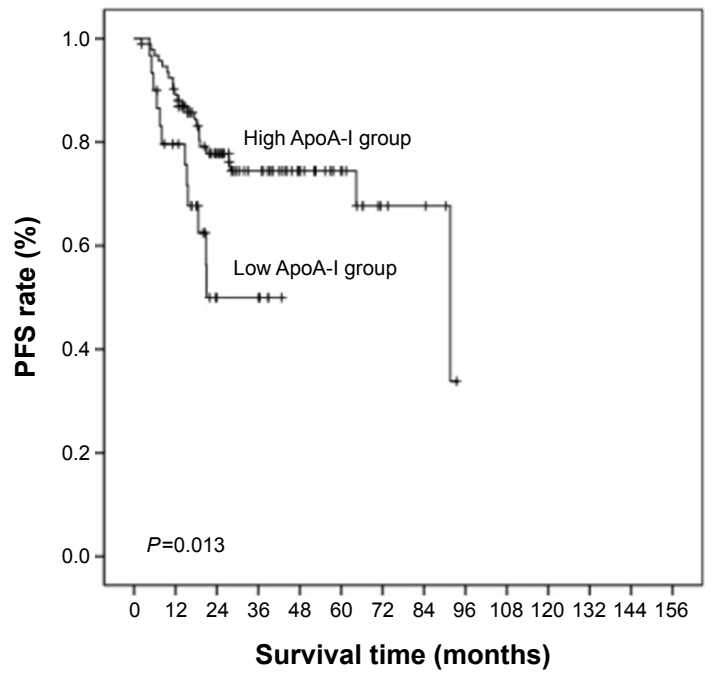

Figure 3 OS and PFS according to ApoA-I level ( $\leq 0.95$ vs $>0.95 \mathrm{~g} / \mathrm{L})$ in the subgroups.

Notes: Kaplan-Meier plots of OS (A) and PFS (B) for subgroups with low IPI scores of 0-I. OS (C) and PFS (D) for subgroups with low PIT scores of 0-I. OS (E) and PFS (F) for subgroups with low KPI scores of $0-1$.

Abbreviations: ApoA-I, apolipoprotein A-I; IPI, International Prognostic Index; KPI, Korean Prognostic Index; OS, overall survival; PFS, progression-free survival; PIT, Prognosis Index for peripheral T-cell lymphoma, unspecified. 
prospective designs are warranted to confirm our findings and to better illustrate the mechanisms underlying the relationship between ApoA-I level and prognosis in ENKTL disease.

\section{Acknowledgments}

The authors would like to thank the patients and their families and all investigators, including the physicians, nurses, and laboratory technicians, for their contributions to this study. This research did not receive any grant support.

\section{Disclosure}

The authors report no conflicts of interest in this work.

\section{References}

1. Harabuchi Y, Yamanaka N, Kataura A, et al. Epstein-Barr virus in nasal T-cell lymphomas in patients with lethal midline granuloma. Lancet. 1990;335(8682):128-130.

2. Au WY, Ma SY, Chim CS, et al. Clinicopathologic features and treatment outcome of mature T-cell and natural killer-cell lymphomas diagnosed according to the World Health Organization classification scheme: a single center experience of 10 years. Ann Oncol. 2005;16(2):206-214.

3. Lee J, Suh C, Park YH, et al. Extranodal natural killer T-cell lymphoma, nasal-type: a prognostic model from a retrospective multicenter study. J Clin Oncol. 2006;24(4):612-618.

4. Au WY, Weisenburger DD, Intragumtornchai T, et al. Clinical differences between nasal and extranasal natural killer/T-cell lymphoma: a study of 136 cases from the International Peripheral T-Cell Lymphoma Project. Blood. 2009;113(17):3931-3937.

5. Kim TM, Lee SY, Jeon YK, et al. Clinical heterogeneity of extranodal NK/T-cell lymphoma, nasal type: a national survey of the Korean Cancer Study Group. Ann Oncol. 2008;19(8):1477-1484.

6. Kim SJ, Kim K, Kim BS, et al. Phase II trial of concurrent radiation and weekly cisplatin followed by VIPD chemotherapy in newly diagnosed, stage IE to IIE, nasal, extranodal NK/T-cell lymphoma: Consortium for Improving Survival of Lymphoma study. J Clin Oncol. 2009;27(35):6027-6032.

7. Yamaguchi M, Kwong YL, Kim WS, et al. Phase II study of SMILE chemotherapy for newly diagnosed stage IV, relapsed, or refractory extranodal natural killer (NK)/T-cell lymphoma, nasal type: the NK-Cell Tumor Study Group study. J Clin Oncol. 2011;29(33): 4410-4416.

8. Vose J, Armitage J, Weisenburger D. International peripheral T-cell and natural killer/T-cell lymphoma study: pathology findings and clinical outcomes. J Clin Oncol. 2008;26(25):4124-4130.

9. Chim CS, Ma SY, Au WY, et al. Primary nasal natural killer cell lymphoma: long-term treatment outcome and relationship with the International Prognostic Index. Blood. 2004;103(1):216-221.

10. Gallamini A, Stelitano C, Calvi R, et al. Peripheral T-cell lymphoma unspecified (PTCL-U): a new prognostic model from a retrospective multicentric clinical study. Blood. 2004;103(7):2474-2479.

11. Rodriguez J, Conde E, Gutierrez A, et al. The results of consolidation with autologous stem-cell transplantation in patients with peripheral T-cell lymphoma (PTCL) in first complete remission: the Spanish Lymphoma and Autologous Transplantation Group experience. Ann Oncol. 2007; 18(4):652-657.

12. Huang JJ, Jiang WQ, Lin TY, et al. Absolute lymphocyte count is a novel prognostic indicator in extranodal natural killer/T-cell lymphoma, nasal type. Ann Oncol. 2011;22(1):149-155.

13. Au WY, Pang A, Choy C, Chim CS, Kwong YL. Quantification of circulating Epstein-Barr virus (EBV) DNA in the diagnosis and monitoring of natural killer cell and EBV-positive lymphomas in immunocompetent patients. Blood. 2004;104(1):243-249.
14. Li YJ, Li ZM, Xia Y, et al. Serum C-reactive protein (CRP) as a simple and independent prognostic factor in extranodal natural killer/T-cell lymphoma, nasal type. PLoS One. 2013;8(5):e64158.

15. Cai Q, Luo X, Liang Y, et al. Fasting blood glucose is a novel prognostic indicator for extranodal natural killer/T-cell lymphoma, nasal type. Br J Cancer. 2013;108(2):380-386.

16. Brewer HB Jr, Fairwell T, LaRue A, Ronan R, Houser A, Bronzert TJ. The amino acid sequence of human APOA-I, an apolipoprotein isolated from high density lipoproteins. Biochem Biophys Res Commun. 1978; 80(3):623-630.

17. Yui Y, Aoyama T, Morishita H, Takahashi M, Takatsu Y, Kawai C. Serum prostacyclin stabilizing factor is identical to apolipoprotein A-I (Apo A-I). A novel function of Apo A-I. J Clin Invest. 1988;82(3): 803-807.

18. Wang X, Dai S, Zhang Z, et al. Characterization of apolipoprotein A-I as a potential biomarker for cholangiocarcinoma. Eur J Cancer Care. 2009; 18(6):625-635.

19. Nosov V, Su F, Amneus M, et al. Validation of serum biomarkers for detection of early-stage ovarian cancer. Am J Obstet Gynecol. 2009; 200(6):639.e631-e635.

20. Jiang R, Yang ZH, Luo DH, et al. Elevated apolipoprotein A-I levels are associated with favorable prognosis in metastatic nasopharyngeal carcinoma. Med Oncol. 2014;31(8):80.

21. Cheng T, Dai X, Zhou DL, Lv Y, Miao LY. Correlation of apolipoprotein A-I kinetics with survival and response to first-line platinum-based chemotherapy in advanced non-small cell lung cancer. Med Oncol. 2015; 32(1):407.

22. Kim TM, Park YH, Lee SY, et al. Local tumor invasiveness is more predictive of survival than International Prognostic Index in stage I(E)/II(E) extranodal NK/T-cell lymphoma, nasal type. Blood. 2005; 106(12):3785-3790.

23. A predictive model for aggressive non-Hodgkin's lymphoma. The International Non-Hodgkin's Lymphoma Prognostic Factors Project. N Engl J Med. 1993;329(14):987-994.

24. Cheson BD, Horning SJ, Coiffier B, et al. Report of an international workshop to standardize response criteria for non-Hodgkin's lymphomas. NCI Sponsored International Working Group. J Clin Oncol. 1999; 17(4): 1244.

25. Adult Treatment Panel. Third Report of the National Cholesterol Education Program (NCEP) Expert Panel on Detection, Evaluation, and Treatment of High Blood Cholesterol in Adults (Adult Treatment Panel III) final report. Circulation. 2002;106(25):3143-3421.

26. Chen KL, Liu YH, Li WY, et al. The prognostic nutritional index predicts survival for patients with extranodal natural killer/T cell lymphoma, nasal type. Ann Hematol. 2015;94(8):1389-1400.

27. Lin ZX, Bai B, Cai QC, et al. High numbers of tumor-associated macrophages correlate with poor prognosis in patients with mature $\mathrm{T}$ - and natural killer cell lymphomas. Med Oncol. 2012;29(5):3522-3528.

28. Wang L, Wu-Xiao ZJ, Chen XQ, Zhang YJ, Lu Y, Xia ZJ. Hepatitis B virus infection correlates with poor prognosis of extranodal natural killer/T cell lymphoma. Leuk Lymphoma. 2015;56(4):936-941.

29. Lee J, Park YH, Kim WS, et al. Extranodal nasal type NK/T-cell lymphoma: elucidating clinical prognostic factors for risk-based stratification of therapy. Eur J Cancer. 2005;41(10):1402-1408.

30. Balkwill F, Mantovani A. Inflammation and cancer: back to Virchow? Lancet. 2001;357(9255):539-545.

31. Coussens LM, Werb Z. Inflammation and cancer. Nature. 2002; 420(6917):860-867.

32. Van Lenten BJ, Wagner AC, Anantharamaiah GM, et al. Apolipoprotein A-I mimetic peptides. Curr Atheroscler Rep. 2009;11(1):52-57.

33. Kim J-S, Chang J-H, Yu H-K, et al. Inhibition of angiogenesis and angiogenesis-dependent tumor growth by the cryptic kringle fragments of human apolipoprotein(a). J Biol Chem. 2003;278(31):29000-29008.

34. Navarro MA, Carpintero R, Acín S, et al. Immune-regulation of the apolipoprotein A-I/C-III/A-IV gene cluster in experimental inflammation. Cytokine. 2005;31(1):52-63.

35. Xu Y, Fang XJ, Casey G, Mills GB. Lysophospholipids activate ovarian and breast cancer cells. Biochem J. 1995;309(Pt 3):933-940. 
36. Eliopoulos AG, Stack M, Dawson CW, et al. Epstein-Barr virus-encoded LMP1 and CD40 mediate IL-6 production in epithelial cells via an NF-kappaB pathway involving TNF receptor-associated factors. Oncogene. 1997;14(24):2899-2916.

37. Ettinger WH, Varma VK, Sorci-Thomas M, et al. Cytokines decrease apolipoprotein accumulation in medium from Hep G2 cells. Arterioscler Thromb. 1994;14(1):8-13.

38. Feng W, Gao X, McClung G, Zhu S, Ishihara K, Brash JL. Methacrylate polymer layers bearing poly(ethylene oxide) and phosphorylcholine side chains as non-fouling surfaces: in vitro interactions with plasma proteins and platelets. Acta Biomater. 2011;7(10):3692-3699.

39. Li ZM, Zhu YJ, Sun J, et al. Serum beta2-microglobin is a predictor of prognosis in patients with upper aerodigestive tract NK/T-cell lymphoma. Ann Hematol. 2012;91(8):1265-1270.

40. Mihou D, Katodritou E, Zervas K. Multiple myeloma staging based on the combination of beta-2-microglobulin and albumin: the role of albumin in the model. Hematology. 2007;12(6):527-531.
41. Roxburgh CS, McMillan DC. Cancer and systemic inflammation: treat the tumour and treat the host. Br J Cancer. 2014;110(6):1409-1412.

42. Wang L, Chi PD, Chen H, Xiang J, Xia ZJ, Zhang YJ. Low level of high-density lipoprotein cholesterol correlates with poor prognosis in extranodal natural killer/T cell lymphoma. Tumour Biol. 2014;35(3): 2141-2149.

43. Su F, Kozak KR, Imaizumi S, et al. Apolipoprotein A-I (apoA-I) and apoA-I mimetic peptides inhibit tumor development in a mouse model of ovarian cancer. Proc Natl Acad Sci. 2010;107(46):19997-20002.

44. Gao F, Vasquez SX, Su F, et al. L-5F, an apolipoprotein A-I mimetic, inhibits tumor angiogenesis by suppressing VEGF/basic FGF signaling pathways. Integrative Biol. 2011;3(4):479-489.
OncoTargets and Therapy

\section{Publish your work in this journal}

OncoTargets and Therapy is an international, peer-reviewed, open access journal focusing on the pathological basis of all cancers, potential targets for therapy and treatment protocols employed to improve the management of cancer patients. The journal also focuses on the impact of management programs and new therapeutic agents and protocols on

\section{Dovepress}

patient perspectives such as quality of life, adherence and satisfaction. The manuscript management system is completely online and includes a very quick and fair peer-review system, which is all easy to use. Visit http://www.dovepress.com/testimonials.php to read real quotes from published authors. 